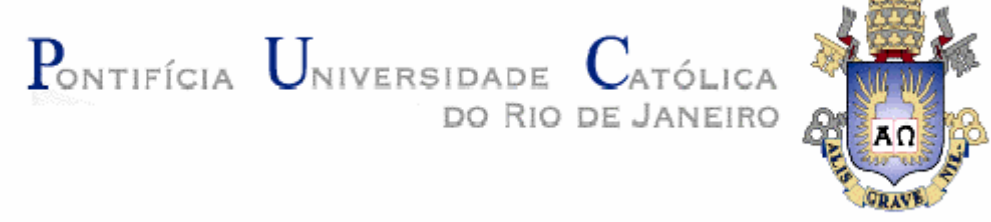

Flavia Nizia da Fonseca Ribeiro

\title{
Internet e Imagem: representações de jovens universitários
}

Tese de Doutorado

\begin{abstract}
Tese apresentada como requisito parcial para obtenção do Título de Doutor pelo Programa de Pós-Graduação em Educação do Departamento de Educação da PUC-Rio.
\end{abstract}

Orientador: Prof ${ }^{\mathrm{a}}$ Maria Apparecida C. Mamede Neves 


\title{
Pontifícia Universidade C atólica $_{\text {a }}$

Flavia Nizia da Fonseca Ribeiro

Internet e Imagem: representações de jovens universitários

Tese apresentada como requisito parcial para obtenção do Título de Doutor pelo Programa de Pós-graduação em Educação do Departamento de Educação do Centro de Teologia e Ciências Humanas da PUC-Rio. Aprovada pela Comissão Examinadora abaixo assinada.

\author{
Prof ${ }^{a}$. Maria Apparecida Campos Mamede Neves \\ Orientadora \\ Departamento de Educação - PUC-Rio \\ Prof ${ }^{a}$ Rosália Maria Duarte \\ Departamento de Educação - PUC-Rio \\ Prof $^{\text {a }}$. Rita Maria de Souza Couto \\ Departamento de Artes e Design - PUC-Rio
}

Profa. Stella Cecília Duarte Segenreich

UCP

Prof. Pier Cesare Rivoltella Universita Cattolica del Sacro Cuore di Milano

Prof. Paulo Fernando C. de Andrade Coordenador Setorial do Centro de Teologia e Ciências Humanas

Rio de Janeiro, 25 de abril de 2008. 
Todos os direitos reservados. É proibida a reprodução total ou parcial do trabalho sem autorização da universidade, da autora e do orientador.

\section{Flavia Nizia da Fonseca Ribeiro}

Flavia Nizia da Fonseca Ribeiro graduou-se em Desenho Industrial - habilitação em Comunicação Visual em 1995, pela Pontifícia Universidade Católica do Rio de Janeiro (PUC-Rio). Obteve o título de Mestre em Design pela PUC-Rio em 2001, com a dissertação "Práticas Pedagógicas em Cursos de Graduação de Design", orientada pela Prof ${ }^{\mathrm{a}}$. Dr $^{\mathrm{a}}$. Rita Maria de Souza Couto. Atua como pesquisadora do Laboratório de Pedagogia do Design, Departamento de Artes e Design /PUCRio, desde 1999, e do Grupo de Pesquisa Jovens em Rede, Departamento de Educação/PUC-Rio, desde 2001. Estágio de doutorado, financiado pela CAPES, e realizado juntamente ao Centro di Ricerca sull'Educazione ai Media all'Informazione e alla Tecnologia (CREMIT) da Università Cattolica del Sacro Cuore di Milano, durante o qual teve a oportunidade de atuar como pesquisadora e consultora. Experiência docente na graduação de Design e Pedagogia, especialização e extensão, Atuação no campo da Educação à Distância onde já coordenou cursos, projetou materiais além de orientar monografias e exercer tutorias. Suas áreas de interesse são: Design, Ensino, Imagem e Tecnologia, nas quais vem desenvolvendo pesquisas e reflexões.

Ficha Catalográfica

\section{Ribeiro, Flavia Nizia da Fonseca}

Internet e imagem: representações de jovens universitários / Flavia Nizia da Fonseca Ribeiro ; orientadora: Maria Apparecida C. Mamede Neves. - 2008. $181 \mathrm{f}$; $30 \mathrm{~cm}$

Tese (Doutorado em Educação)-Pontifícia Universidade Católica do Rio de Janeiro, Rio de Janeiro, 2008.

Inclui bibliografia

1. Educação - Teses. 2. Imagem. 3. Representação. 4. Internet. 5. Alfabetismo visual. 6. Jovens universitários. I. Neves, Maria Apparecida C. Mamede. II. Pontifícia Universidade Católica do Rio de Janeiro. Departamento de Educação. III. Título. 
Deus que permitiu que este trabalho fosse realizado, dando os incentivos justos e colocando as pessoas certas no meu até quando eu achava que não estava mais na rota certa. 


\section{Agradecimentos}

A CAPES pela concessão da bolsa de estudos aqui no Brasil e na Itália e ao CNPq que financiou a pesquisa Jovens em Rede na qual o meu trabalho esteve inserido.

A minha incansável orientadora, Apparecida Mamede, que foi acima de qualquer coisa, companheira, apoiando meus delírios, me tirando as inseguranças. Que Deus a conserve por muito tempo! Obrigada é pouco por tudo que você tornou possível.

Aos professores Celso Wilmer, Fátima Queiroz, Rivoltella, Rosália Duarte, Stella Segenreich, Rita Couto, que se dispuseram a ler e avaliar este trabalho. Aos professores Miguel de Faria e Fernando Vidal pelas breves e preciosas consultorias.

Ao grupo de pesquisa Jovens em Rede por enlouquecerem com meus quadradinhos coloridos e ainda assim comprarem a idéia, além do imenso apoio nas parcerias das pesquisas! Vocês são um auxílio mais que luxuoso!

Ao pessoal da secretaria do Departamento de Educação, em especial Geneci, Janaína e Neiva, que me socorrem com sorrisos, abraços e favores desde muito antes do doutorado ser uma realidade. Vocês foram, são, e sempre serão muito especiais para mim!

A todas as pessoas que fizeram doações de revistas, e que o cansaço do final da escrita não me permite enumerá-las uma a uma, para que eu realizasse os grupos focais.

A Antonio Mamede, plácida companhia nos retiros acadêmicos, pela calma confortante e pelos brindes em nome desta tese. Vicky Maia pela incansável disponibilidade de explicar e re-explicar conceitos psi e semióticos, e Fernando 
Maia, pela 'escovação' dos números e por transformas os quadradinhos coloridos em maravilhosos gráficos. A Verinha que carinhosamente fazia com que o retiro fosse um oásis!

A minha turma do doutorado, em especial: Elis Bernado, pequena grande e doce amiga; Fernando Gouvêa, o homem do bom humor, visão otimista, meu companheiro da aventura 'francês'; Bernadete Strang, pela amizade sincera.

Um super, ultra, mega, infinito obrigada as amigas Ana Valéria Figueiredo e Stella Pedrosa, dizer as razões da gratidão seria ser injusta pois não há como contá-las. Obrigada por vocês existirem na minha vida!

A família LPD, a matriarca desta família, Ritinha, por tudo que você é e representa,não importando a distância em que esteja.

A todos os meus amigos, que não são poucos, sem exceção. Desculpem pelas eventuais ausências, obrigada pela compreensão.

A todos os 'biscoitos' do Studio Creamcrackers pelas infindáveis respostas às minhas dúvidas, um beijo especial a Soninha que desde meu mestrado assume o papel do ‘anjo que caiu do céu’!

Aos queridíssimos André - que ilustrou este trabalho -, Aline - que tornou real a parte gráfica - e Tito, pelo infindável, incansável e incondicional apoio sempre.

A querida amiga Mônica Fantin, pelo apoio delicado e substancial nas dúvidas e inseguranças da experiência italiana.

A Antonio Bottino, un vero maestro, e oggi un amico, che mi ha insegnato le prime parole in italiano, e ha aiutato a rendere possibile parte di questa ricerca.

A Prof. Pier Cesare Rivoltella per le domande giuste nei tempi giusti, per le opportunità alla Cattolica di Milano, per la fiducia e, soprattutto, per l'amicizia. Ringrazio anche la sua famiglia per l' accoglienza e per i bei pranzi e le partite di calcio!

A famiglia Friso per l' accoglienza, a famiglia Ghilotti per i bei momenti familiari che sono stati molto speciali, il vostro affetto ha fatto sembrare la distanza dai miei familiari, più piccola.

Tutte le care persone della Cattolica - del dipartimento di pedagogia, del CEPaD, del Master Comunicazione e formazione e del Corso di Perfezionamento in Media Education - per gli sguardi curiosi, per le attenzioni nelle lezioni e oltre le lezione, per ogni sorriso, ogni 'buon giorno!', ogni cappuccino al bar, ogni pranzo, ogni 'buon appetito!', ogni chiacchiere, ecc. Speciale ringraziamenti nelle persone di: Professore Cesar Scurati; Agnese Tirabassi; Andrea Garavaglia; Anna Ragosta; Caterina Pedretti; alle Chiara Marazzi, Rizzi e Valmachino; Damiano Felini; Elena Valdameri; Elsa Zoffi; Fabrizio Catalano; Francesca Musetti; Giulio Tosone (essooo!); Gloria Sinini; Irene Sturlese; Laura Comaschi; Laura Giuliaci e suo Davide; Lorenza Ruggiero; Max Massimiliano; Monica Crotti; Roberta Nascimbembe; Simona Ferrari; Vera Carminati; Silvio Premoli. Grazie immenso per ogni piccola cosa. Le piccole cose danno senso alla nostra vita. 
Priscilla Molaro, e suo Tito, Silvia Inventi Solari e Suzanna Lera, le ragazze con cui ho diviso l'apartamento, bollete, tv, chiacchiere, spese e ecc.

Juliana Hu, Liliane Chaves, Marlise Santos, Olavo Bessa le persone che hanno reso Milano più brasiliana per me.

A Ivan Castellame (gne!) per l'amicizia, per gli sms che sono arrivati con le parole certe, per la bella passeggiata in moto e per le altre nel mondo del virtuale.

A caro Prof. Paolo Ardizzone, per ogni sorriso lento e per essere il più bravo studente delle mie lezione di portoghese!

A Davide Tosi per la attenzione, sei grande! A Tony, hai reso la mia vita più dolce con la tua personalità e con i tuoi pasticcini.

A Magda Pischetola per tutto, per la buona e brava persona che sei e che mi emoziona sempre. A Chiara Checcini per la compagnia al giro in Portogallo.

A Claudia Clerici e suo Lorenzo, per i bellissimi momenti San Remo, quelli degli Scigula e tanti altri sempre pieni di affetti.

Alla fine però non meno importante, specialissimi ringraziamenti di cuore:

Alessandra Carenzio e Carlo, vocês são uns amores, pessoas mais que especiais que moram no meu coração! Chiara Friso e Michele Aglieri, miei cuccioli, cari amici, sorella e fratello, compagni, miei scemi preferiti. Grazie di cuore per tutto, sarebbe poco dire per ringraziare ogni momenti con voi. Siete persone più che importanti, fondamentali, già eterni nella mia storia. Non c'è 12 senza 88 , non c'è latte senza biscotto, non c’è Italia senza di voi.

Aos tios, tias, primos, primas e etc. obrigada por estarem sempre onde estão mesmo quando eu mesma não posso estar! Obrigada por entenderem as ausências mesmo quando muitas vezes as razões para elas pareciam exageradas. Por fim, a minha avó, meus pais e irmão, por me aturarem e serem a base do que sou. Amo vocês! 


\section{Resumo}

Ribeiro, Flávia Nizia da Fonseca; Mamede-Neves, Maria Apparecida Campos. Internet e Imagem: representações de jovens universitários. Rio de Janeiro, 2008, 181 p. Tese de Doutorado - Departamento de Educação, Pontifícia Universidade Católica do Rio de Janeiro.

Esta tese tem como objeto central as representações, por palavras ou imagens, que jovens recém ingressos na universidade fazem da Internet. $\mathrm{O}$ trabalho tomou como premissa básica a imagem como representação visual, seja ela desenho ou texto, apoiando-se em autores como Ave Appiano, Erwin Panofsky, Jacques Aumont, Lorenzo Vilches, W. J. T. Mitchell, entre outros, que pensam a imagem e texto como uma unidade. A pesquisa, desenvolvida com alunos da PUC-Rio, usou um desenho metodológico que se desenvolveu em dois momentos sucessivos. No primeiro momento, foi realizado um estudo de caráter exploratório sobre o uso e representações de diferentes mídias e, em particular, da Internet, por parte dos universitários. O segundo momento foi constituído por uma investigação de caráter qualitativo, tendo como estratégias de coleta de dados o grupo focal de vivências proposto por Fern e Morgan e a entrevista. Os dados colhidos no questionário aplicado no primeiro momento da investigação possibilitaram o levantamento das representações mais freqüentes da Internet, numa amostra de 995 jovens, representações essas que foram usadas como disparadores da discussão havida dentro da primeira parte do segundo momento, qual seja a dos grupos focais cujos membros foram escolhidos dentro de uma amostra intencional.. A segunda parte da dinâmica realizada nos grupos permitiu obter, também, produções em desenho ou colagens realizadas por cada um dos componentes dos grupos focais, representando o que o jovem espera da Internet. Todas essas representações, expressas por palavras e/ou por imagens foram analisadas, primeiramente, de forma específica dentro de cada conjunto. Tendo como referência estudos como os de Derrick de Kerckhove, Lev Manovich, Pier Cesare Rivoltella, Roger Fidler, Sherry Turkle, Steven Johnson, foi realizado igualmente a análise do cruzamento de todos os dados empíricos recolhidos, análise esta que se deu em torno de alguns eixos temáticos: a naturalização da Internet, a sua representação simbólica em termos de velocidade, informação, comunicação , diversão ,globalização. A Internet está muito presente no cotidiano desses jovens, o que pode ser verificado através da grande sintonia que demonstram ter, além de uma postura crítica em relação à Internet, tomada como uma unidade. Aspectos positivos e negativos com relação ao seu uso, suas vantagens, desvantagens, limitações, utilidade, e até seus perigos foram constatados. Outra constatação desta pesquisa foi a evidente mudança de hábitos cotidianos, escolares ou sociais para esses universitários, ratificando a importância do 'alfabetismo visual', nos termos propostos por Donis Dondis, para esta nova forma de construção do conhecimento.

\section{Palavras-chave}

Imagem; representação; Internet; alfabetismo visual; jovens universitários. 


\section{Abstract}

Ribeiro, Flávia Nizia da Fonseca; Mamede-Neves, Maria Apparecida Campos (Advisor). The Internet and Image: representations by young university students. Rio de Janeiro, 2008, 181 p. Thesis - Departamento de Educação, Pontifícia Universidade Católica do Rio de Janeiro.

This thesis aims mainly at the representations, through words or images, which college freshmen make of the Internet. This paper has as a basic premise image as a visual representation, be it a drawing or a text, based on authors such as Ave Appiano, Erwin Panofsky, Jacques Aumont, Lorenzo Vilches, W. J. T. Mitchell, among others, who understand image and text as one unit. The research, developed with PUC-Rio students, used a methodological design developed over two successive periods. Firstly, an exploratory study was carried out on the use and the representations of different media, and, in particular, of the Internet, on the part of university students. Secondly, a qualitative investigation was carried out which used as data collecting strategies the focal experimentation groups proposed by Fern and Morgan and interviews. The data obtained through the query applied at the first stage of the investigation enabled eliciting the most frequent representations of the Internet, from a sample of 995 youths, which were used to trigger discussions carried out during the first part of the second stage, that of the focal groups whose members were chosen by intentional sampling. The second part of the group dynamics allowed the obtaining productions such as drawings or collages made by each and every member of the focal groups, representing what youths expect from the Internet. All these representations, expressed through words and/or images, were first analyzed specifically within each set. Based on studies such as those by Derrick de Kerckhove, Lev Manovich, Pier Cesare Rivoltella, Roger Fidler, Sherry Turkle, and Steven Johnson, an analysis was carried out of the crossing of all the empirical data collected; such analysis revolved around some thematic axes: the naturalization of the Internet, its symbolic representation in terms of speed, information, communication, entertainment, globalization, labyrinth and invisibility . The Internet is very present in the daily lives of these youths, which can be verified by the great attunement which they seem to have, besides a critical posture before the Internet, all taken as a unit. Positive and negative aspects of its use, its advantages and disadvantages, its limitations, its usefulness, even its dangers were elicited. This research also verified the evident change of daily school and social habits for these university students, confirming the importance of 'visual literacy', as defined by Donis Dondis, for this new manner of knowledge construction.

\section{Key-words}

Image; representation; the Internet; visual literacy; young university students. 


\section{Sumário}

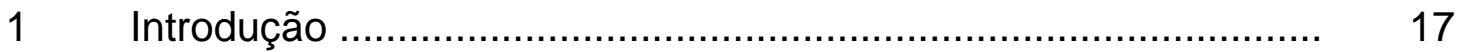

2 Forma, imagem e representação .............................................. 26

2.1 Imagem e representação ....................................................... 26

2.1.1 A contribuição da Psicanálise ................................................... 30

2.1.2 A contribuição da escola da Gestalt ......................................... 39

2.2 Forma da realidade e forma da representação ............................ 41

$2.3 \quad$ Imagem e signo ............................................................. 51

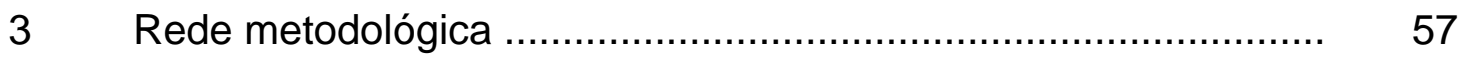

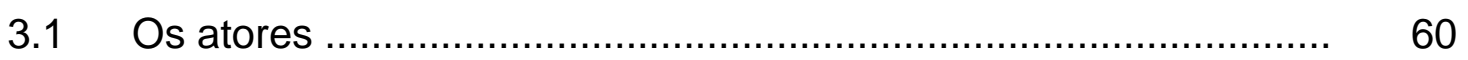

3.2 Os instrumentos ............................................................. 62

3.3 Procedimentos adotados …………………......................... 73

4 Jovens universitários: quem são, o que fazem e o que pensam .. 75

4.1 Do questionário ................................................................. $\quad 75$

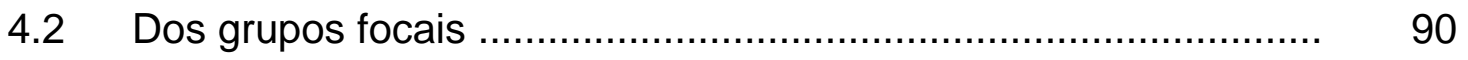

4.2.1 Grupo GF1 ...................................................................... 90

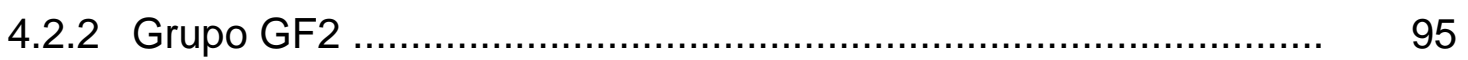

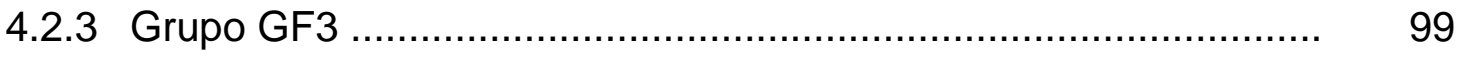

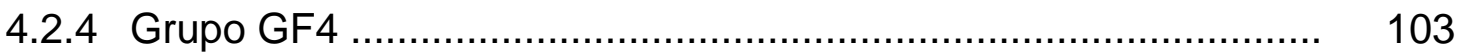

$5 \quad$ Imagens e representações da Internet ...................................... 108

5.1 Características, simbologias e metáforas …………………........ 129

5.1.1 Naturalização .................................................................. 130

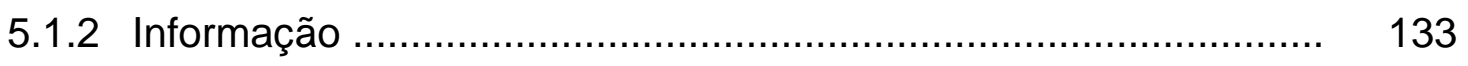

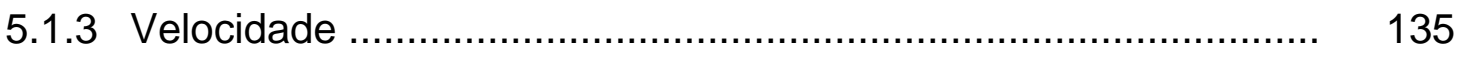

5.1.4 Globalização ................................................................... 137

5.1.5 Comunicação ................................................................ 139 
5.1.6 Entretenimento ........................................................... 140

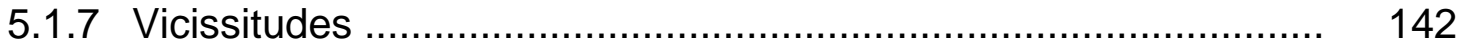

5.1.8 Invisibilidade ..................................................................... 144

5.1.9 Navegação ...................................................................... 147

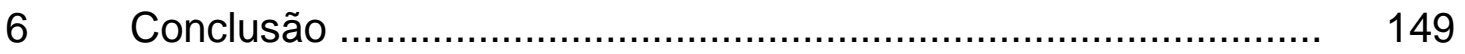

$7 \quad$ Referências Bibliográficas........................................................ 153

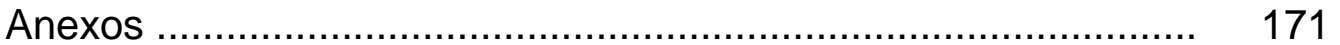




\section{Lista de figuras}

Figura 1 Panorama conceitual prévio da pesquisa ............................. 19

Figura 2 Diagrama de inserção da pesquisa em um contexto maior ... 21

Figura 3 Mapa conceitual das possíveis abordagens do tema ............ 22

Figura 4 Diagrama imagem mental vs. imagem real .......................... 29

Figura 5 Sistema Percepção-Consciência segundo Freud .................. 32

Figura 6 Representação de mente humana de Herbart ....................... 32

Figura $7 \quad$ O uso do bloco mágico .................................................. 33

Figura 8 Diagrama psicológico de uma apresentação da palavra ....... 38

Figura 9 Pintura rupestre de aproximadamente 12 mil anos Parque Nacional Serra da Capivara, Piauí, Brasil ................ 42

Figura 10 Pinturas estilizadas da Caverna de Castillo de Monfragüe, Cáceres, Espanha - Idade do Bronze .................................. 43

Figura 11 Caverna das Mãos na Patagônia, aproximadamente 9 mil anos .................................................................... 43

Figura 12 Jardins do Palácio de Mateus - Vila Real, Portugal ............... 43

Figura 13 Jardim Barroco .............................................................. 44

Figura 14 Jardins do Palácio Nacional de Queluz, Sintra, Portugal ........ 44

Figura 15 Macaco desenhado com sulcos na Terra pela população Nazca, Peru. - civilização pré-colombiana ............................ 44

Figura 16 Candelabro Nazca, Peru ................................................. 44

Figura 17 Detalhe das linhas de um dos desenhos Nazca, Peru ........... 45 
Figura 18 Realidade, percepção e interpretação

Figura 19 Modelo de análise semiótica da imagem .....

Figura 20 Diagrama do contexto de inserção da pesquisa

Figura 21 Exemplo de codificação para tabulação do questionário ...... 63

Figura 22 Configuração da tabulação do questionário 63

Figura 23 Configuração da tabulação da pergunta 36 do questionário ... 65

Figura 24 Imagem 01 do grupo focal 68

Figura 25 Imagem 02 do grupo focal 68

Figura 26 Imagem 03 do grupo focal 69

Figura 27 Imagem 04 do grupo focal 69

Figura 28 Imagem 05 do grupo focal 69

Figura 29 Imagem 06 do grupo focal 69

Figura 30 Imagem 07 do grupo focal 69

Figura 31 Imagem 08 do grupo focal 69

Figura 32 Imagem 09 do grupo focal 69

Figura 33 Imagem 10 do grupo focal 69

Figura 34 Imagem 11 do grupo focal 69

Figura 35 Fotos da realização de um dos grupos focais 73

Figura 36 Fotos da realização de um dos grupos focais 73

Figura 37 Fotos da realização de um dos grupos focais 73

Figura 38 Exemplo de diagrama de disposição da realização de um dos grupos focais

Figura 39 Exemplo de ficha de registro das produções dos grupos focais

Figura 40 Exemplo de um fotolog

Figura 41 Exemplo de uma notícia online ilustrada por um vídeo ... 83

Figura 42 Exemplo de uma pagina de um site de relacionamentos 84

Figura 43 Exemplo de uma chat em um programa de comunicação .......

Figura 44 Produção individual de Fo24 do GF1 109

Figura 45 Produção individual de Ro34 do GF1 110

Figura 46 Produção individual de Fa34 do GF2

Figura 47 Produção individual de Vo22 do GF1

Figura 48 Produção individual de Ga33 do GF2

Figura 49 Produção individual de Po18 do GF2 . 
Figura 50 Produção individual de Pa19 do GF2 ….............................. 115

Figura 51 Produção individual de Ta42 do GF2 ................................. 116

Figura 52 Produção individual de Ta18 do GF2 ................................. 117

Figura 53 Produção individual de Va00 do GF2 ................................ 118

Figura 54 Produção individual de Mo19 do GF3 ……......................... 119

Figura 55 Produção individual de Do19 do GF3 .................................. 120

Figura 56 Produção individual de Ro17 do GF3 .................................. 121

Figura 57 Produção individual de Ro17 do GF3 ................................. 122

Figura 58 Produção individual de Aa29 do GF1 ................................. 124

Figura 59 Produção individual de Ga24 do GF2 .................................. 125

Figura 60 Produção individual de Ca19 do GF4 ................................. 126

Figura 61 Produção individual de Ro20 do GF4 …….......................... 126

Figura 62 Produção individual de Aa19 do GF4 ................................. 128

Figura 63 Ilustração do ideal do Projeto Xanadu ................................... 135

Figura 64 Desenho de uma criança lugoslava para o Projeto Kidlink ..... 139

Figura 65 A banda de Moebius ……............................................ 145

Figura 66 Símbolos borromeanos em detalhes .................................. 146

Figura 67 Símbolos borromeanos em detalhes ................................... 146

Figura 68 Símbolos borromeanos em detalhes .................................. 146

\section{Lista de gráficos}

Gráfico 1 Distribuição das representações da Internet sobre as categorias criadas ............................................................ 68

Gráfico 2 Distribuição dos estudantes por idade ................................ 76

Gráfico 3 Meio de comunicação mais usado no tempo livre ............... 76

Gráfico 4 Quantidade de televisão que assiste desde que começou a ter Internet em casa .................................................... 78

Gráfico 5 Quantidade de música que escuta desde que começou a 79 
ter Internet em casa

Gráfico 6 Quantidade de leitura desde que começou a ter Internet em casa

Gráfico 7 Quantidade de vezes que sai com os amigos desde que começou a ter Internet em casa

Gráfico 8 Meio de comunicação mais usado para se manter informado 80

Gráfico 9 O melhor suporte para estudo 81

Gráfico 10 Para que usam a Internet

Gráfico 11 Quais serviços disponíveis na Internet usados com mais regularidade

Gráfico 12 Respostas da categoria identificativo 86

Gráfico 13 Respostas da categoria emotivo 86

Gráfico 14 Respostas da categoria cognitivo 86

Gráfico 15 Respostas da categoria relacional 87

Gráfico 16 Comparação Internet x computador 87

Gráfico 17 Comparação Internet x livro 88

Gráfico 18 Comparação Internet $x$ televisão 88

Gráfico 19 Histograma das 1123 respostas em 219 tipos diferentes ..... 89

Gráfico 20 Histograma das respostas com 10 ou mais ocorrências

Gráfico 21 Que fazem ao mesmo tempo que estão conectados a Internet

Gráfico $22 \mathrm{Na}$ Internet existe a possibilidade de viver coisas impossíveis, como fingir ser alguém que não é, inventar coisas etc 


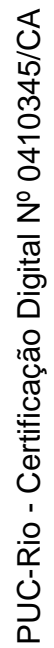

Represento logos existo.. 